\title{
DESIGN OF SUPER CAVITATION SUBMARINE NOSE THAT SUSTAINS UNDER HIGH HYDRAULIC PRESSURE
}

\author{
D. Vignesh ${ }^{1}$, Sohan Saha ${ }^{2}$ \\ ${ }^{1}$ Student, Department of Mechanical Engineering, SRM University, Tamil Nadu, India \\ ${ }^{2}$ Student, Department of Mechanical Engineering, SRM University, Tamil Nadu, India
}

\begin{abstract}
The main aim of this research work is to design an effective model of submarine nose system that provides suitable cavitation which in turn helps in increasing the speed and sustainability of the body under water. The research work also involves the design of a rigid nose against deformation caused by the pressure and penetration action using the geodesic dome structures. Though already many works have been done in achieving super cavitation in submarine body, this paper mainly concentrates on the nose design to sustain the hydraulic pressure and provision of cavitation vents around the nose.
\end{abstract}

Keywords: super cavitation, turbulence free high speed motion, rigid submarine nose design, geodesic dome. ****

\section{INTRODUCTION}

The modern day transportation of both men and materials are done through conventional modes of transportation. Hence in present scenario, the transportation through oceans needs to be developed in order to meet the requirements of the present demands. For these above reasons, there is a huge demand of innovation are needed in these sectors. The primary goal in sub water transportation is to increase the speed of transportation. To meet this requirement, super cavitation should be achieved in the submarines to reduce the hydrodynamic drag and turbulence. In these days there are various research works are carried on to achieve super cavitation, also in such high speed the nose of the body should be resistant to deformation caused by hydraulic forces and action of pressure. For these above reasons certain modifications are to be done in the design of the nose and those parameters are discussed in this paper work.

\section{PROBLEMS FACED BY THE}

\section{CONVENTIONAL MODEL}

The conventional design has a wider nose and has a steeper tail structure, although this design is well suited to sustain under high hydraulic pressure, it greatly affects the speed. Hence the conventional model suffers from a major disadvantage of hydraulic drag and increased separation of the layers of fluid which in turn reduces the speed of the body.

Since the conventional model has very good rigidity against the pressure and buoyancy forces, the model is preferred since yet. If the super cavitation is to be introduced in the system, certain modifications in the design are to be done. These modifications affect the sustainability properties of the body under hydraulic forces. For this reason the nose of the body should be designed according to the implementation of the cavitation.

\subsection{Modified New Model}

The new model design has two modifications over the conventional model. They are as follows;

i. Implementation of super cavitation in the body

ii. Modification of nose geometry to sustain the fluid pressure

These two modifications are the main factors that play a major role in overcoming the drawbacks of the conventional model. The super cavitation in the body reduces hydraulic drag of the fluid by avoiding the contact of surface of the body with the fluid and thereby reduces the formation of boundary layers which in turn reduces hydraulic friction factor. By reducing the friction caused by the hydraulic layer the speed of the body can be increased.

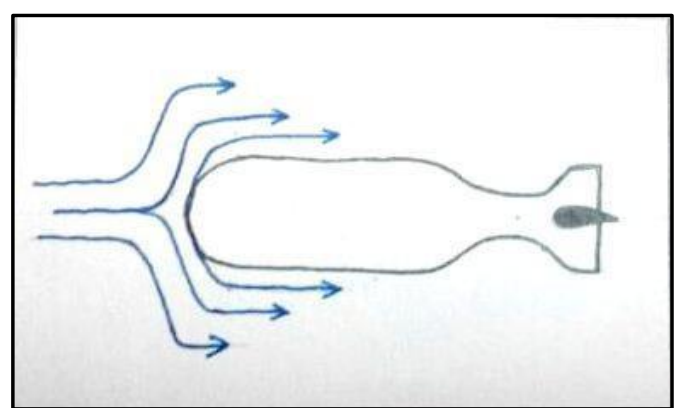

Fig 2.1 Drag force faced by conventional vessel

Though this model has a good advantage over speed of the submarine, it too suffers from heavy impact of hydraulic forces at that much amount of speed under water. The front area of the submarine is largely affected due to this hydraulic force; hence the special nose design provides the solution to this problem. 


\section{RECTIFICATION OF PROBLEMS BY} REPLACING THE CONVENTIONAL MODEL WITH NEW DESIGN

The major aim of this paper work is to implant the artificial super cavitation system in the warfare submarines and to modify the same to rectify the problems faced. Some of the major drawbacks which are rectified by replacing the conventional design with the new one are as follows;

1. Pressure and stress sustainability of the frontier part of the vessel.

2. Operational speed of the vessel under high hydraulic pressure.

3. Drag force implied over the surface of the vessel due to working fluid.

4. Propeller driving power under extreme knots.

These drawbacks are rectified by modifying the preexisting conventional vessel design and the design consideration and justification of the modifications are discussed in the following topics

\subsection{Parameters to be considered}

The design of the nose portion depends upon various parameters, by considering the positive \& negative effects of the modification and by analyzing proportional \& inverse parameters; the design is to be done. The following are the parameters that are to be considered before initiating the designing process of the nose portion;

a. Nose geometry,

b. Working pressure,

c. Operating speed.

\subsubsection{Nose Geometry}

The nose geometry is commonly paraboloidal in nature, but to with stand high hydraulic forces and pressure, a higher thickness wall should be used. This action increases the weight which in turn reduces the speed. To avoid this, nose geometry should be modified in such a way that it withstands the pressure and other hydraulic forces.

The nose tip and the successor portions are to be designed like a "Geodesic dome", since the geodesic dome is made of triangles; they are very rigid in nature. This action eliminates the usage of thick shells by replacing them with triangular skeletons. So the deformation rate with respect to the forces is very less and the portion becomes light in weight.

\subsubsection{Working Pressure}

Most of the submarines are designed on the basis of the working pressure and test pressure parameters. Human workers on the submarine under hydraulic pressure would suffer from many psychological problems if the pressure inside the vessel is equal to the hydraulic pressure acting outside the body. This is because oxygen under high pressure becomes dense which has toxic effect over the human beings, hence the body of the submarine should be designed in such a way that it with stands the pressure difference.

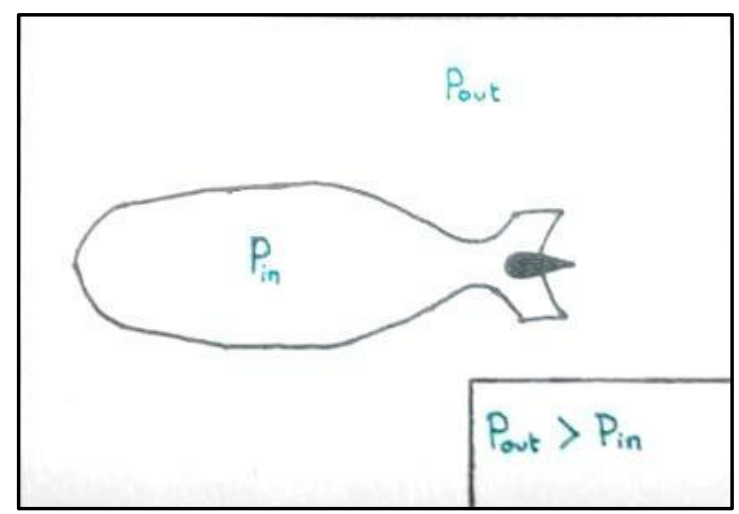

Fig 3.1 Pressure difference in and outside the vessel

Generally the inside pressure of the vessel should be in one atmospheric pressure $(100 \mathrm{kPa})$ and the working depth of the vessel will be greater than 1000 feet which will be equivalent to 31 atmospheric pressure $(3100 \mathrm{kPa})$. To with stand this much pressure and to sustain the same stability under super cavitation effect, the nose geometry should be augmented with additional skeleton structure with less weight. This condition is achieved by the above specified dome design.

\subsubsection{Operating Speed}

The major aim of providing super cavitation in the submarines is to increase the speed of the vessel, so with respect to the requirement and the vessel sizing the operational speed varies. The common submarines operates under the speed range of 40 45 knots, but recent research has revealed that the unmanned warfare submarines can reach the speed up to 200 knots with the help of super cavitation. Though many research works are done in this field, still the implementation of this concept in the working model which resembles the theoretical predictions is still on progress and yet to be achieved, this is because of the problems that are concentrated over the vessel by the working atmosphere of the body.

The present research work clearly illustrates all the drawbacks and possible remedies for the above mentioned problems, in further topics.

\section{NEW MODEL DESIGN}

The new model design of the super cavitation augmented vessel involves the modifications in the nose part, external hull geometry, air intake vanes and control system. The modifications that are made in the existing model and new innovations integrated in the model are discussed below; Initially, the nose model has been modified in such a way that it withstands the hydraulic forces and pressure that is implied over the vessel by the surrounding fluid. The conventional model has a smooth nose which is made of hardened material which is of high density or of high cost to withstand the forces, but in this case a special nose model is 
to be used to serve the purpose. The new design utilizes the simple geodesic dome design to meet the requirement.

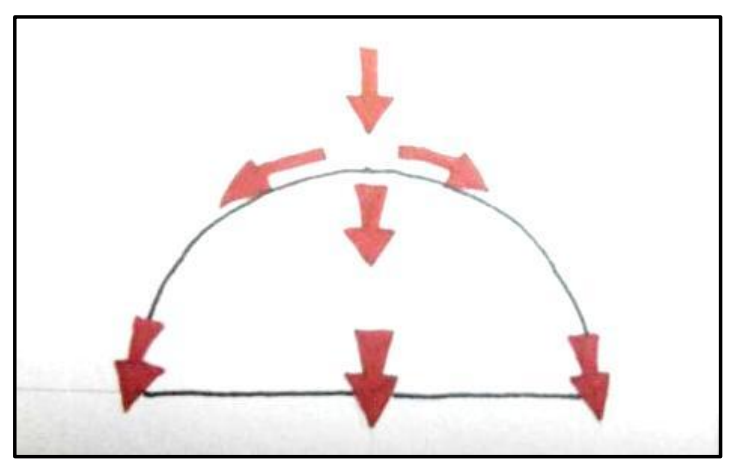

Fig 4.1 Force distribution over Geodesic dome nose

Since the geodesic dome uses the combination of triangles to form the shape, it provides a rigid link at the corners which is prone to deformation under the pressure. Based on the size of the vessel the geodesic nose design varies, as the diameter scales from small $(3 \mathrm{~m})$ to large $(9 \mathrm{~m}+)$ the dome design is chosen from $1 \mathrm{~V}$ to $6 \mathrm{~V}$ respectively.

Secondly, the pump jet propulsion is chosen over the conventional propeller to eliminate the boiling of fluid at the rear part due to reduction in pressure caused by the propeller rotation. This too helps in reduction of drag and sound produced by the diffusion of bubbles, which are caused by the low pressure boiling effect. Since the specified propeller uses the fluid jet effect to generate thrust, it needs only a small amount of energy to produce thrust which shows that this propeller has greater efficiency than the conventional one.

Since the noise produced is very much minimum, there occurs a reduction in sonar signature which may be said as an additional advantage of this kind of propulsion system.

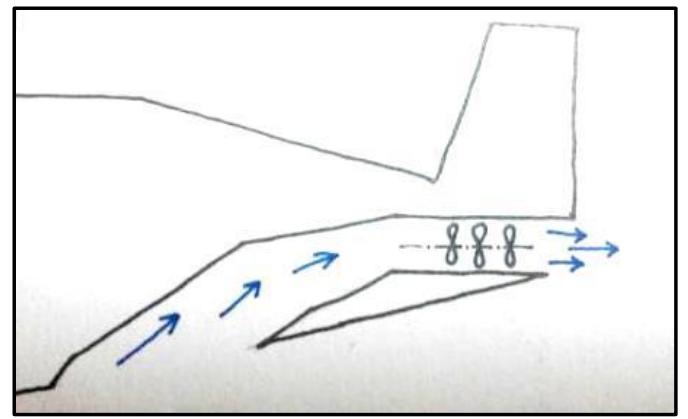

Fig 4.2 Representation of Pump jet propulsion

Finally, the exterior geometry is modified to reduce the drag. Changing the conventional exterior and designing a new body to resist the drag consumes a lot work and time, but modifying some particular portion itself is sufficient to serve the purpose. In that case, the nose portion and the middle portion are to be smooth, hence the additional projections and irregularities are to be eliminated. Since the cavitation initiates at the nose and terminates with a huge area, the projections and other necessary irregularities can be placed at the rear part of the vessel.

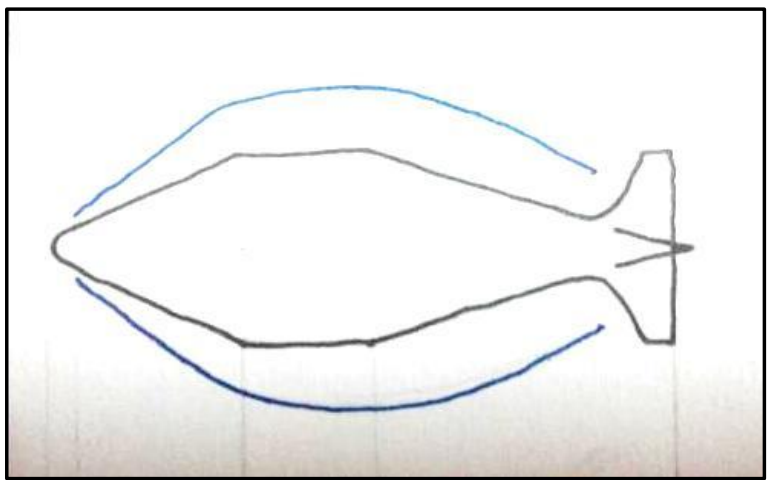

Fig 4.3 Nose design to reduce drag

This modification contributes much to the reduction of hydrodynamic drag at higher knots.

\section{DESIGN EQUATIONS}

The basic design equations are similar to that of the conventional design procedures, some of the design equation that are to be modified and augmented to the design process are discussed below;

\subsection{Cavitation Number}

The cavitation number $(\sigma)$ plays a major role in determining the shape and size of the artificial super cavitation in submarines. The parameters influencing and determining the cavitation number are clearly explained using the following relation;

$$
\sigma=\frac{2\left(P_{i}-P_{c}\right)}{\rho v_{i}^{2}}
$$

where,

$\mathrm{P}_{\mathrm{i}}$-Instantaneous pressure

$\mathrm{P}_{\mathrm{c}}-$ Cavitation pressure

$\rho$-Working medium density

$\mathrm{v}_{\mathrm{i}}$ - Operating velocity

As the super cavitation number decreases, the cavitation dimension increases. Since the relation between them is inversely proportional, for large submarines the number $\sigma$ should be minimum and vice versa.

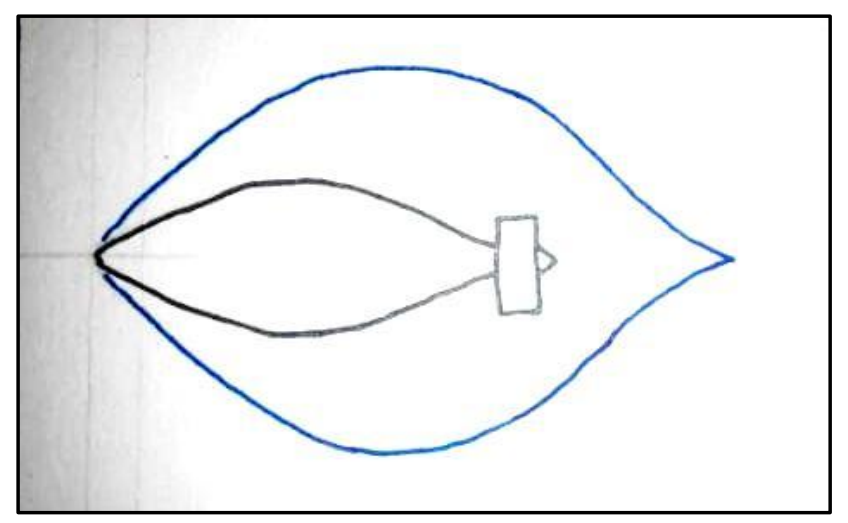

Fig 5.1 Representation of cavitation over vessel 
The factors that decrease the value of the cavitation number are explained below,

- Operating velocity of the body may be a direct factor that affects the cavitation number. If the velocity increases, then the number decreases and hence the cavity dimension increases.

- Pressure difference between the surrounding to the cavity should be less. This is achieved at depth near sea level, as the depth increases the pressure difference becomes maximum and hence the number $\sigma$.

On considering these parameters the cavitation number can be determined and so can the shape and size of the artificial cavity around the vessel.

\subsection{Cavity Ventilation using Gases}

There are three methods to use the gases to create artificial cavitation, they are Sedov scheme for cavitation, Gas supply from nose portion and Gas supply past nose portion. Of all the above mentioned techniques, the Gas supply past nose portion is an effective technique to create artificial super cavitation and hence this technique is used in this model.

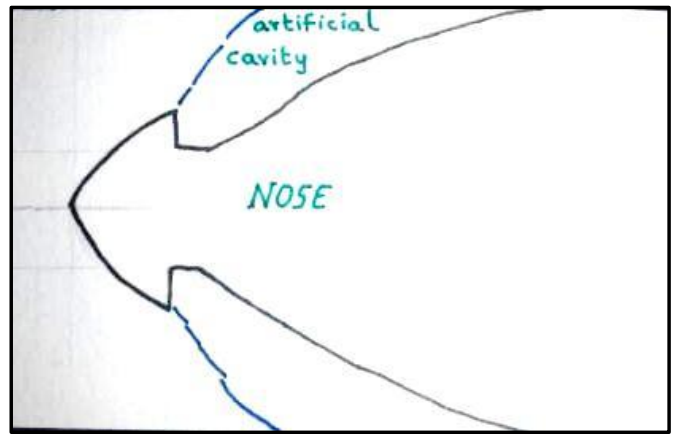

Fig 5.2 Gas supply past nose portion

The chosen technique works as follows, initially the nose portion rams the fluid at the front portion of the vessel and followed by this action the gases are blown out of the rear nose cavity and hence creating an artificial cavitation around the vessel. This technique involves the "Gas supply coefficient (Q')" to determine the gas blow rate from the rear nose cavity. The influencing parameters of the gas supply coefficient are shown using the following relation.

$$
Q^{\prime}=\frac{Q}{v_{i} D_{n}^{2}}
$$

where,

$\mathrm{Q}$ - Volumetric gas rate at cavity pressure

$\mathrm{D}_{\mathrm{n}}-$ Cavity diameter

The parameter Q' has unit $\mathrm{m}^{3} / \mathrm{sec}$ and it is to be noted that the gases are to be ejected from the rear part of the nose through jet air ejection and the velocity of the gases are to be greater than that of the vessel velocity to flow past the body in opposite stream to it. The following equation gives the relation between the gas velocity $\left(\mathrm{v}_{\mathrm{g}}\right)$ and the vessel velocity $\left(\mathrm{v}_{\mathrm{i}}\right)$.

$$
v_{g}>v_{i} \sqrt{(\sigma+1) \frac{\rho}{\rho_{g}}} \quad \begin{aligned}
& \begin{array}{l}
\text { where } \\
\rho_{\mathrm{g}}-\text { Density of cavitation } \\
\text { gas }
\end{array}
\end{aligned}
$$

The above discussed two relation determines the formation of artificial cavitation around the body.

\subsection{Nose Geometry Design}

The nose part is made with Geodesic dome geometry to eliminate the frame level deformation under high operating pressure. Based on the size of the vessel the diameter of the nose varies, and the variation is from $1 \mathrm{~V}$ to $6 \mathrm{~V}$ as discussed. Most of the submarines have dimensions in same range so the design of Geodesic dome of range $3 \mathrm{~V}$ is discussed in here,

$$
R_{D}=\frac{\text { strut length }}{\text { strut factor }} \quad \begin{aligned}
& \text { where, } \\
& \mathrm{R}_{\mathrm{D}}-\text { Nose radius }
\end{aligned}
$$

For a $3 \mathrm{~V}$ geodesic dome we have three strut $(\mathrm{A}, \mathrm{B}, \mathrm{C})$ and hence three strut factors $\left(A^{*}, B^{*}, C^{*}\right)$.

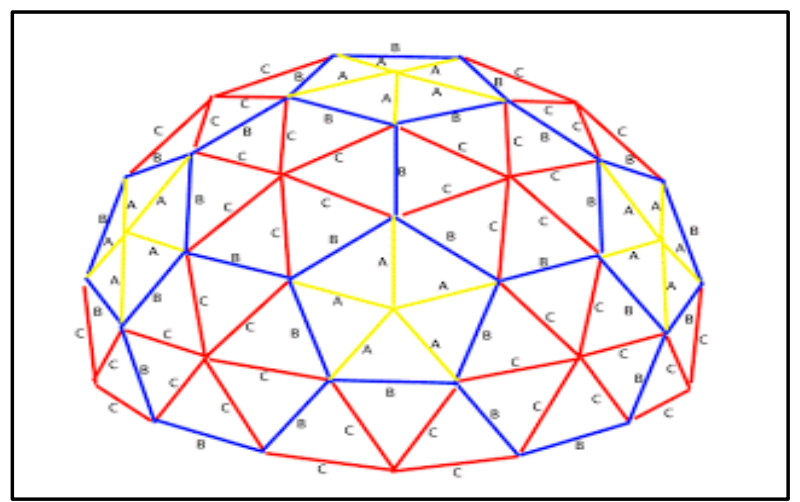

Fig 5.3 Geodesic dome (3V) construction

The strut factors are the constant values that are obtained from practical experimental results. The predefined strut factor values for the $3 \mathrm{~V}$ dome are as follows;

\begin{tabular}{|l|l|}
\hline Strut & Strut factors \\
\hline A & 0.3486 \\
\hline B & 0.4035 \\
\hline C & 0.4124 \\
\hline
\end{tabular}

Using the above mentioned constants, an effective dome structures for the vessel nose can be designed based on its nose radius.

\section{COMPARISON EQUATIONS}

The difference between the conventional vessel design and the new design can also be expressed in terms of their property and design equations. This approach clearly illustrates the differences and proves the sustainability of the new design under super cavitation effect. Some of the major comparison equations are discussed below; 


\subsection{Rigidity of the Nose Geometry}

The rigidity of the nose portion plays a major role in the vessel design under cavitation effect, as discussed in above topics. In this case the individual motion of the links from which the nose is constructed, should be zero.

$$
M=\sum_{i=1}^{j} f_{i}-3 \quad \begin{aligned}
& \text { where, } \\
& \mathrm{M}-\text { Motion } \\
& \mathrm{j}-\text { no of joint }
\end{aligned}
$$

Here, for geodesic dome whose geometry is completely made of triangles, the number of joints is 3 and $f_{i}$ is 3 and hence the $\mathrm{M}$ becomes zero. This shows that there is no individual frame deformation (ie motion $\mathrm{M}$ ).

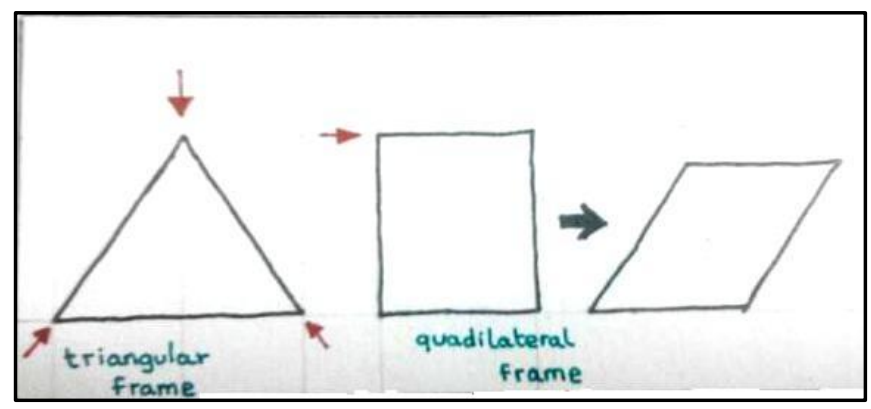

Fig 6.1 Comparison of frames

Now, in the case of conventional geometry, the meshed surface of the nose shows that it is made of small squares and rectangles. For these quadrilaterals, the number of joints are 4 and $f_{i}$ is 4 and on substituting the values in the above equation, we get $M=1$. This shows that there will be a deformation in the geometry since there is a motion $\mathrm{M}$.

\subsection{Cavitation Drag}

The cavitation drag plays a major role in the operating speed of the vessel. In conventional vessels where the artificial super cavitation is not implanted, the drag force increases. This parameter is clearly explained with the following relation.

$$
F_{D}=(0.5) \rho v^{2} C_{D} A
$$

where,

$F_{D}$ - Drag Force

$\rho$ - Density of the medium

$\mathrm{v}-$ Velocity of the vessel

$\mathrm{C}_{\mathrm{D}}$ - Drag coefficient

A - Area of interaction

In case of cavitation the working medium will be gases whose density $(\rho)$ is very minimal when compared to liquids, hence the drag force falls proportionately w.r.t to the density of the working medium. When the drag force reduces, the velocity of the body increases proportionately. In case of the conventional vessels there is no cavitation and hence the body has saline water as its working medium whose density $(\rho)$ is greater than the gases, hence the drag force is greater than the previous one. This shows that the velocity of the conventional model is much lesser than the new vessel with super cavitation effect.

\subsection{Power required to overcome Drag Force}

When the vessel experience a drag force, certain amount of power is required to overcome the drag force and this energy is supplied from the propeller of the vessel.

$$
P_{D}=F_{D} v
$$

where,

$\mathrm{P}_{\mathrm{D}}$ - Power required

Since the $F_{D}$ is minimal when the working medium has less density, which is seen when the vessel uses super cavitation concept, the power required to overcome the drag force is minimal at this case than the conventional model.

\subsection{Efficiency of the propeller}

The efficiency of the propeller plays a major role in effective utilization of the engine power at high operating speed. The propeller efficiency is defined as the ratio between the propulsive power out, to the input shaft power. The input shaft power may also be referred as $\mathrm{P}_{\mathrm{D}}$ at extreme cases, to overcome the drag.

$$
\eta_{p}=\frac{\text { Propulsive power out }}{\text { Input shaft power }}
$$

where,

$\eta_{\mathrm{p}}-$ Propeller efficiency

From the previous relation, it is known that the input shaft power is less for the super cavitation implanted body which implies the efficiency of the vessel is greater than the conventional one.

\section{APPLICATIONS OF THE MODEL IN CURRENT SITUATIONS}

The present day submarines are widely used for defence purposes and are used only for under water survey and safeguarding the boundaries. If the super cavitation concept is implanted in the submarines, then the vessel can be used in various scenarios and their applications are discussed below;

- The super cavitation provides high speed operational range to the vessel and hence this concept improves the utility of the vessel in extensive defence purposes.

- Through super cavitation, the time of travel in submarines gets reduced and hence this improves the underwater transportation.

- Since the nose design in modified to sustain the force implied by the super cavitation effect, the vessel can also used for deep sea dwelling purposes where the pressure range is very high. 
These are some of the applications of the super cavitation implanted vessel in current scenario.

\section{PROBLEMS FACED AND REMEDIES}

Some of the major problems faced by the super cavitation implanted vessel and their remedies are as follows;

\subsection{Problems Faced}

1. Concentrated stresses on the front nose,

2. Heat produced over the surface of the body due to hydrodynamic heating,

3. Supply of gases for super cavitation,

4. Controlling the vessel at high operating speed,

5. Complexity in building the vessel.

\subsection{Remedies}

1. Concentrated stresses on the front nose: Due to the motion of the vessel in forward direction, a large amount of stresses will be induced in the nose portion at such a high velocity. This contributes much to the deformation or damage of the nose of the vessel, if the vessel is made with thick layer of metal then the weight at the front portion increases and at this scenario the balancing of the body is to be considered. To meet these both problems an effective nose design with the help of geodesic dome geometry is to be done, which solves both the problems faced under the working scenario.

2. Heat produced due to hydrodynamic heating: Under extreme velocities the hydrodynamic heating takes place all over the surface area of the body, though the effect of hydrodynamic heating is much lesser when compared to the aerodynamic heating, it contributes much to the thermal stresses concentration over the vessel. This factor can be controlled by eliminating the additional projections over the surface of the vessel, in other words the whole body should be smooth and it should be designed in such a way that there is no obstruction for the fluid flow over the surface of the vessel.

3. Supply of gases for super cavitation: In most of the conventional submarines with super cavitation setup, a separate gas chamber is utilized to store the gases and use it during the required stage. This concept is best suited for conventional vessels with partial super cavitation effect, but for the prolong application of the concept a large amount of gases are required and the storage of such a huge amount of gases is an impossible task. To meet this problem a couple of vents are to be augmented to the body to intake the atmospheric air and utilize the same after compressing it. This system eliminated the need of huge gas storage tank in the vessel.

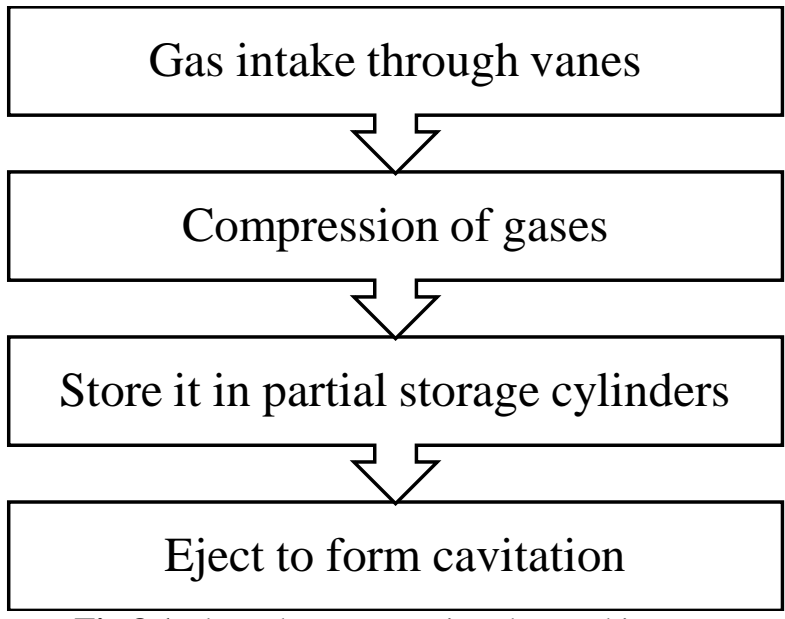

Fig 8.1 Flow chart expressing the working process

The partial storage cylinder said here is a miniature storage tank that partially stores the compressed gases.

4. Controlling the vessel at high operating speed: The vessel has very high momentum and it is difficult to change its predefined pathway at the required instant, this is due to the hydraulic forces acting over the outer surface of the vessel. To change the path, the fins are to be rotated, but under the hydraulic forces the fins are subjected to deformation. The only remedy of the problem is to minimize the requirement of sudden changes in the pathway of the vessel. In other words, an obstruction free pathway is to be predetermined before initiating the operation, if any temporary obstruction approaches, then it is to be ramified by the nose portion.

5. Complexity in building the vessel: Though the new model super cavitation vessel has more complex vents and gaseous intake system, building of such a kind of vessel is a hard task. Additional to the vents, the whole body should be aerodynamically fit to avoid hydrodynamic heating; this makes the building process even more complex. Hence the unique solution of this problem is to work from the scratch rather than modifying an existing vessel to meet the requirements. This leaves a pathway to design and manufacture the unique parts as per the requirements, to fit inside/over the vessel.

\section{ADVANTAGES AND DISADVANTAGES OF}

\section{THE MODEL}

Some of the pros and cons of the new model super cavitation implanted vessel is listed below;

\subsection{Advantages}

1. The new model vessel can travel at high knots with the help of artificial super cavitation.

2. The vessel can work under extreme pressure, since the frame level rigidity is very high in this case.

3. The skin friction of the vessel's surface area is much minimum since the fluid around the body is gas instead of liquid. 
4. No need to have a gas storage tank in this vessel since it takes the atmospheric air through vanes for the cavitation purpose.

5. The propeller shaft driving power is much lesser than that of the conventional vessel.

6. The Hydrodynamic heat produced over the body will be removed through the cavitation effect.

These are some of the advantages of the new model design over the conventional design.

\subsection{Disadvantages}

Though the system is designed in such a way that it rectifies the problems in the conventional model, this new model has its own disadvantages. Some of them are discussed below;

1. It is difficult to maintain the cavitation number at a constant value throughout the process.

2. The vessel may face change in acceleration (jerk) at high operating speed, since the cavity may change its shape w.r.t cavity number $(\sigma)$.

3. Manufacturing the air inlet vanes has its own complexity level.

\section{CONCLUSION AND FUTURE WORKS}

The presented approach introduces the new system for under water transportation and utilization of the geodesic dome geometry for designing the front nose of the vessel to survive under such a large pressure and stresses, which is a new concept in this area. Some of the future developments that are to be made in this super cavitation system are as follows:

1. Further modifications are to be done on the body so that the whole body shows reluctance over the deformation caused by the work area pressure and stresses induced.

2. The wall thickness of the vessel is to be reduced to increase the velocity of the body; this topic needs a lot of research work to be carried on in the fields of material technology.

3. To design a unique guidance and engine control system to control the vessel at such a high speed under water.

4. To design an effective gaseous intake system, to provide adequate supply of gases for the cavitation system, this topic involves the complete study of the atmospheric air flow inside the suction duct of the vessel.

\section{REFERENCES}

[1] John Allison, "Marine Waterjet Propulsion", SNAME Transactions, Vol. 101, 1993.

[2] Dr. R.K. Bansal, "A Textbook of Fluid Mechanics and Hydraulic Machines", Ch 1, 3, 9, 15.

[3] Dr. D. Padmanaban \& Dr. P. Aruna. "Design Data, Data Book of Engineers".

[4] Samuel, Andrew; Weir, John. "Introduction to engineering design: modeling, synthesis and problem solving strategies "(2ed.). Oxford, England: Butterworth.

[5] http://www.dtic.mil/dtic/tr/fulltext/u2/p012080.pdf

[6] http://authors.library.caltech.edu/25019/1/chap5.htm
[7] https://www.defensetech.org/2009/11/17/supercavitation-and-the-truth/

[8] http://www.popularmechanics.com/military/research/ a21572/supercavitation-penn-state-researchsubmarines/ 